\title{
UNA MIRADA AL RELATO DE LA HUMANIDAD Y EL PANORAMA DEL DESARROLLO SUSTENTABLE EN LOS ALBORES DEL TERCER MILENIO
}

\section{A LOOK AT THE STORY OF HUMANITY AND THE PANORAMA OF SUSTAINABLE DEVELOPMENT AT THE DAWN OF THE THIRD MILLENNIUM}

Natividad Covarrubias - Tovar ${ }^{1}$

\begin{abstract}
${ }^{1}$ Mexicana. Profesora Titular C. Universidad de Guadalajara. Centro Universitario de Ciencias Económico Administrativas. Departamento de Ciencias Sociales y Jurídicas. Periférico Norte 799, Núcleo Universitario Los Belenes, CP. 45100 Zapopan, Jal.; México Tel: 3770-3300 Extensión 25513. Email: natividad@cucea.udg.mx
\end{abstract}

\begin{tabular}{|l|l}
\hline Recibido: 09.10.18 & Aprobado: 03.05.19
\end{tabular}

DOI:10.15517/isucr.v20i41.38817

Resumen: El abordaje multidimensional del medio ambiente desde las diferentes ciencias o disciplinas ha generado en los distintos escenarios o sedes, un sinnúmero de reflexiones y debates registrados en un universo de documentos, como fieles testimonio de las conceptualizaciones y el cambio de percepción del ambiente, aunado a ello la reorientación de los modelos de desarrollo han forjado un cambio de paradigma, en el que los involucrados: individuos, empresas, organizaciones y gobiernos, deben asumir nuevos compromisos bajo la actuación consciente y de mejora continua, donde la trasparencia, equidad, ética, eficiencia, responsabilidad, pero sobre todo la voluntad, constituyen el motor de un nuevo desarrollo. Como respuesta a este nuevo enfoque en materia de la administración del medio ambiente emergieron nuevas instituciones y organismos en busca de dar respuesta a la problemática del deterioro ambiental, desafortunadamente el esfuerzo ha sido entorpecido por la falta de continuidad de los programas de protección y conservación, manifestados principalmente en un desgaste de recursos económico, humano y hasta ecológico reflejados en el desarrollo pasivo del país y sus actividades productivas.

Hasta hace relativamente poco tiempo se asumía que el papel del desarrollo era solo generar capital, visión que actualmente no es suficiente ni aceptable, el desarrollo ahora implica la consideración de dimensiones básicas: económica, social y ambiental y las complementarias como la tecnología y la política. Se requiere, además, de cambios no solo en los procesos productivos, sino en los hábitos de consumo y en consecuencia en la oferta de nuevos productos y la modificación de sistema de gestión y políticas, que no solo mitiguen el deterioro ambiental, sino que prevengan y lo minimicen.

Palabras claves: Desarrollo; Medio ambiente; Políticas públicas; Estado; 


\begin{abstract}
:
The purpose of this article is to make an analysis of the notion of development. Until relatively recently it was assumed that the role of development was only to generate capital, a vision that is currently not sufficient or acceptable, development now involves the consideration of basic dimensions: economic, social and environmental.
\end{abstract}

Key words: Development; Environment; Public policies; State;

\title{
Introducción
}

El desarrollo humano ha sido influido por su entorno no obstante que el hombre crea lo contrario, el medio ambiente no es solo un escenario para las actividades humanas es el elemento para su subsistencia. Medio ambiente y desarrollo sustentable han sido hilvanados por la historia del hombre, haciendo de ellos un polinomio indisoluble y esencial en la querella ambiental entre los involucrados. Cualquier estilo de desarrollo, aunque no fuera así adjetivado, debe ser obligadamente sustentable, dado el equilibrio entre hombre naturaleza que se requiere.

El medio ambiente puede ser abordado desde diversas aristas como la economía, los recursos naturales, el entorno de los seres vivos, la biología, la ingeniería, la agronomía, la religión, la política, la restauración, el progreso de las naciones o el escenario de la vida... pero cual fuera la perspectiva pareciera que el concepto de medio ambiente se limita al dominio y adopción de diversos términos que construyen un argot y que quienes lo dominan se les llama ambientalistas, baste entonces mencionar: contaminación, composta, capa de ozono, agenda XXI, energía renovable, huella ecológica, cambio climático, bonos carbono o los menos pronunciables, clorofluorocarbonados, fracking, input, output... además si se agrega el maridaje de términos como stress o resiliencia de la psicología, capacidad de carga de la agricultura y sostenibilidad de la silvicultura ${ }^{1}$ o externalidad de la economía... se dice que se habla de medio ambiente. Ello ha pasado reiteradamente de acuerdo a la corriente ideológica en turno como el naturismo, el conservacionismo, el ecologismo o el ambientalismo y ¿por qué no? inclusive el amarillismo ambiental.

Por lo tanto para analizar el panorama del desarrollo humano y lo que nos relata la naturaleza tiene que repasarse desde distintos planos: por un lado las Revoluciones como antecedente detonador de las reorientaciones del desarrollo, y por otro los escenarios, las publicaciones, las instituciones y el marco legal, elementos cuyo registro ya adquiere dimensiones

El término "sostenibilidad" fue creado en 1713 por Hans Carl von Carlowitz en el marco de la explotación forestal, ante la escasez de madera motivada por la destrucción de los bosques. Este concepto denota la explotación de un bosque mediante la tala de una cantidad de madera igual a la que el propio bosque puede reponer, de manera que el bosque sea capaz de regenerarse una y otra vez, nunca llegando a ser deforestado totalmente. La razón principal de su creación fue asegurar el abastecimiento de materia prima, en vista de la escasez de madera existente a nivel suprarregional. 
enciclopédicas pero baste dar una mirada sobre lo más sobresaliente, para formularse una panorámica de la situación actual de la asociación desarrollo y medio ambiente.

\section{Las revoluciones}

Dichas corrientes, anteriormente mencionadas, han sido a su vez detonadas por las revoluciones humanas: Agrícola (Siglo XVIII mediados del siglo XIX); Industrial (segunda mitad del siglo XVIII en Gran Bretaña y que se extendiera décadas después a una buena parte de Europa Occidental y Estados Unidos); Demográfica (Elevado incremento de la población humana, consecuencia de la Revolución Industrial); Económica (súbito cambio de las condiciones en que se lleva a cabo la producción distribución y consumo de bienes y servicios, que particularmente se aplica a los cambios tecnológicos, por lo que suele identificarse también como revolución tecnológica, aunque el concepto es más amplio, también se utiliza la expresión para referirse a las dos primeras grandes transformaciones que han merecido el nombre de Revolución Económica: la Revolución neolítica y la Revolución Industrial de los siglos XVIII y XIX.); Verde (Entre 1940 y 1970 en Estados Unidos) y Tecnológica (desde la segunda mitad del siglo XX). Esta última hoy todavía vigente (Historia Universal 2014, subpágina: 1)

\section{Los escenarios}

Para exponer los debates y actos de compunción humana, numerosas han sido las instancias o sedes mundiales como Berna ${ }^{2}$, Francia ${ }^{3}$ Estocolmo, Tbilisi, Río de Janeiro, Canadá, Nueva York, Johannesburgo, Kioto... donde proliferaron las buenas intenciones plasmadas en acuerdos mundiales como la Agenda XXI, Rio + 5, Rio + 10, Rio + 20, Protocolo de Kioto, Carta de la Tierra, Eco 92. así mismo, se produjeron documentos y publicaciones que han servido como marco de referencia y de registro del acontecer del desarrollo humano, que

1913, Conferencia de Berna Suiza convocada por Sarazin, presidente de la Liga Suiza para la Protección de la Naturaleza, la primera conferencia registrada en la historia sobre la protección de la Naturaleza. (Paisajes naturales). que se cristalizaría años más tarde 1948 en la Unión Internacional para la conservación de la Naturaleza y de sus Recursos (UIPN), organización independiente que agrupa a centenares de otras organizaciones públicas y privadas, al mismo tiempo que convoca sus famosas conferencias internacionales.

31968 (Setiembre). París (Francia). Conferencia Intergubernamental de Expertos sobre una Base científica para el uso racional y conservación de la Biosfera. "Conferencia de la Biosfera". Comienza a gestarse el concepto de desarrollo sustentable. Las primeras bases del concepto de Reserva de Biosfera derivan de esta conferencia. 
desde principios del siglo XX fueron forjando un hito en la toma de conciencia universal sobre las necesidades de la humanidad de replantear su estilo de desarrollo.

\section{Las publicaciones}

La problemática ambiental ha sido un tema inspirador de lo más prolífero, en la actualidad se cuenta ya con un inconmensurable número de publicaciones, por lo que se mencionan solo algunas de las más relevantes a nivel global.

Tabla 1: Publicaciones más relevantes a nivel global en materia de medio ambiente y desarrollo

\begin{tabular}{|c|c|c|c|}
\hline Año & Titulo & Autor & Comentario \\
\hline 1948 & $\begin{array}{l}\text { Nuestro } \\
\text { planeta } \\
\text { saqueado, } \\
\text { (Our } \\
\text { plundered } \\
\text { planet) }\end{array}$ & $\begin{array}{l}\text { Fairfield } \\
\text { Osborn }\end{array}$ & $\begin{array}{l}\text { La primeras publicaciones consideradas precursoras } \\
\text { de la consternación ambiental y del debate sobre el } \\
\text { agotamiento de los recursos publicadas en } 1948\end{array}$ \\
\hline 1948 & $\begin{array}{l}\text { Camino a la } \\
\text { supervivenc } \\
\text { ia }\end{array}$ & $\begin{array}{l}\text { William } \\
\text { Vogt }\end{array}$ & $\begin{array}{l}\text { Este y el libro anterior lanzaron un renacimiento del } \\
\text { maltusianismo en los años } 50 \text { y ejercieron una gran } \\
\text { influencia en Paul Ehrlich }\end{array}$ \\
\hline 1950 & $\begin{array}{l}\text { La bomba } \\
\text { demográfica }\end{array}$ & $\begin{array}{l}\text { Paul } \\
\text { Ehrlich }\end{array}$ & $\begin{array}{l}\text { Su principal argumento es el crecimiento acelerado de } \\
\text { la población mundial con lo que deduce el agotamiento } \\
\text { de los recursos naturales, el impacto irreversible sobre } \\
\text { el medio ambiente, la extensión del hambre en todo el } \\
\text { planeta. }\end{array}$ \\
\hline 1956 & $\begin{array}{l}\text { El papel del } \\
\text { hombre en } \\
\text { el cambio de } \\
\text { la faz de la } \\
\text { Tierra }\end{array}$ & $\begin{array}{l}\text { W. L. } \\
\text { Tomas, }\end{array}$ & $\begin{array}{l}\text { Referente al impacto cada vez mayor del hombre sobre } \\
\text { la naturaleza. }\end{array}$ \\
\hline 1962 & $\begin{array}{l}\text { Primavera } \\
\text { silenciosa } \\
\text { (Silent } \\
\text { spring) }\end{array}$ & $\begin{array}{l}\text { Rachel } \\
\text { Carson }\end{array}$ & $\begin{array}{l}\text { Su público lector traspasó los círculos científicos } \\
\text { habituales y el contenido se trató en numerosos } \\
\text { artículos, foros y secciones de correspondencia de } \\
\text { revistas y diarios, así como en numerosos debates. }\end{array}$ \\
\hline
\end{tabular}




\begin{tabular}{|c|c|c|c|}
\hline & $\begin{array}{l}\text { pesticidas y } \\
\text { plaguicidas } \\
\text { cancerígeno } \\
\text { s, }\end{array}$ & & \\
\hline 1968 & $\begin{array}{l}\text { Artículo } \\
\text { "The } \\
\text { Tragedy of } \\
\text { Commons" }\end{array}$ & $\begin{array}{l}\text { Garrett } \\
\text { Hardin }\end{array}$ & $\begin{array}{l}\text { Publicado en la revista Science, v. } 162 \text { la parábola que } \\
\text { apareció en un folleto poco conocido escrito en } 1833 \text {, } \\
\text { por William Foster Lloyd, Menciona la teoría de La } \\
\text { mano invisible de Adam Smith, y popularizada gracias } \\
\text { a su obra magna, La riqueza de las naciones (1776) }\end{array}$ \\
\hline 1972 & $\begin{array}{l}\text { Los límites } \\
\text { del } \\
\text { crecimiento } \\
\text { es también } \\
\text { conocido } \\
\text { como } \\
\text { Reporte o } \\
\text { libro } \\
\text { Meadows }\end{array}$ & $\begin{array}{l}\text { Donella } \\
\text { Meadows }\end{array}$ & $\begin{array}{l}\text { Informe encargado al MIT por el Club de Roma, } \\
\text { publicado en 1972, poco antes de la primera crisis del } \\
\text { petróleo, y cuya tesis sostiene que si el incremento de } \\
\text { la población mundial, la industrialización, la } \\
\text { contaminación, la producción de alimentos y la } \\
\text { explotación de los recursos naturales se mantiene sin } \\
\text { variación, alcanzará los límites absolutos en la Tierra } \\
\text { durante los próximos cien años. }\end{array}$ \\
\hline 1972 & $\begin{array}{l}\text { El } \\
\text { Manifiesto } \\
\text { para la } \\
\text { sobrevivenc } \\
\text { ia }\end{array}$ & $\begin{array}{l}\text { Edward } \\
\text { Goldsmith } \\
\text { et al, }\end{array}$ & $\begin{array}{l}\text { Afrontaba los problemas del medio ambiente con una } \\
\text { visión holística como única forma de aproximación } \\
\text { válida para la consecución de un objetivo común. }\end{array}$ \\
\hline 1972 & $\begin{array}{l}\text { Una Sola } \\
\text { Tierra de }\end{array}$ & $\begin{array}{l}\text { Barbara } \\
\text { Ward y } \\
\text { Rene } \\
\text { Dubos: }\end{array}$ & $\begin{array}{l}\text { Registra el primer esfuerzo mundial por examinar con } \\
\text { perspectiva universal la problemática del medio } \\
\text { ambiente, no solo desde un punto de vista } \\
\text { estrictamente científico, sino a través de sus aspectos } \\
\text { sociales, económicos y políticos; no sólo en su } \\
\text { manifestación más obvia, la directa contaminación de } \\
\text { la atmósfera y las aguas, sino como un fenómeno } \\
\text { complejo y en las más amplia diversidad de sus } \\
\text { problemas. }\end{array}$ \\
\hline 1973 & $\begin{array}{l}\text { Resilience } \\
\text { and } \\
\text { Stability of }\end{array}$ & $\begin{array}{l}\text { Crawford } \\
\text { Hollingi }\end{array}$ & $\begin{array}{l}\text { Introduce por primera vez el concepto de resiliencia en } \\
\text { la literatura ecológica como una forma para } \\
\text { comprender las dinámicas no lineales así como los } \\
\text { procesos a través de los cuales los ecosistemas se auto- }\end{array}$ \\
\hline
\end{tabular}




\begin{tabular}{|c|c|c|c|}
\hline & $\begin{array}{l}\text { Ecological } \\
\text { Systems }\end{array}$ & & $\begin{array}{l}\text { mantienen y persisten frente a perturbaciones y los } \\
\text { cambios. }\end{array}$ \\
\hline 1973 & $\begin{array}{l}\text { Lo pequeño } \\
\text { es hermoso: } \\
\text { Economía } \\
\text { como si la } \\
\text { gente } \\
\text { importara }\end{array}$ & $\begin{array}{l}\text { E. F. } \\
\text { Schumach } \\
\text { er }\end{array}$ & $\begin{array}{l}\text { Colección de ensayos por el economista alemán } \\
\text { Schumacher. Publicado por primera vez en } 1973 \text {, } \\
\text { críticas de la economía occidental con un público más } \\
\text { amplio durante la Crisis del petróleo de } 1973 \text { y la } \\
\text { aparición del proceso de globalización. }\end{array}$ \\
\hline 1977 & $\begin{array}{l}\text { Guía para } \\
\text { perplejos }\end{array}$ & $\begin{array}{l}\text { E. F. } \\
\text { Schumach } \\
\text { er }\end{array}$ & $\begin{array}{l}\text { Una crítica al materialismo cientificista y una } \\
\text { exploración de la naturaleza y la organización del } \\
\text { conocimiento. }\end{array}$ \\
\hline 1980 & $\begin{array}{l}\text { Overshoot } \\
\text { (Sobrecarga } \\
\text { ), }\end{array}$ & $\begin{array}{l}\text { de William } \\
\text { R. Catton } \\
\text { Jr }\end{array}$ & $\begin{array}{l}\text { Hizo evidente que la naturaleza, en un futuro no muy } \\
\text { lejano, debía instituir procedimientos de bancarrota } \\
\text { contra la civilización industrial. }\end{array}$ \\
\hline 1986 & $\begin{array}{l}\text { World } \\
\text { Resources }\end{array}$ & $\begin{array}{l}\text { Instituto } \\
\text { de Rec. } \\
\text { Mundiales }\end{array}$ & $\begin{array}{l}\text { Es una guía del ambiente mundial publicada de forma } \\
\text { bianual por la WRI por sus siglas en inglés (World } \\
\text { Resources Institute). }\end{array}$ \\
\hline 1998 & $\begin{array}{l}\text { Carta } \\
\text { mundial de } \\
\text { la } \\
\text { naturaleza }\end{array}$ & ONU & $\begin{array}{l}\text { Primer instrumento jurídico del derecho ambiental } \\
\text { internacional, aunque no obligatorio. }\end{array}$ \\
\hline 1987 & $\begin{array}{l}\text { Nuestro } \\
\text { Futuro } \\
\text { Común } \\
\text { (Our } \\
\text { Common } \\
\text { Future): }\end{array}$ & $\begin{array}{l}\text { Comisión } \\
\text { Brundtlan } \\
\text { d (distintas } \\
\text { naciones) }\end{array}$ & $\begin{array}{l}\text { También conocido como Reporte o informe } \\
\text { Brundtland. Enfrenta y contrasta la postura de } \\
\text { desarrollo económico vigente junto con el de } \\
\text { sostenibilidad ambiental con el propósito de analizar, } \\
\text { criticar y replantear las políticas de desarrollo } \\
\text { económico globalizador, reconociendo que el avance } \\
\text { social se está llevando a cabo a un costo } \\
\text { medioambiental alto. }\end{array}$ \\
\hline $\begin{array}{l}1990- \\
1992\end{array}$ & $\begin{array}{l}\text { Nuestra } \\
\text { Propia } \\
\text { Agenda }\end{array}$ & $\begin{array}{l}\text { CEALC- } \\
\text { CEPAL }\end{array}$ & $\begin{array}{l}\text { Informe de la Comisión Económica para América } \\
\text { Latina y el Caribe preparatoria para la Cumbre de la } \\
\text { Tierra o Río 92, agenda común para los países de } \\
\text { América Latina, donde se recogen los lineamientos } \\
\text { para la formulación de políticas públicas con } \\
\text { orientación ambientalista (BID-PNUD). }\end{array}$ \\
\hline
\end{tabular}




\begin{tabular}{|c|c|c|c|}
\hline 1992 & $\begin{array}{l}\text { Más allá de } \\
\text { los Límites } \\
\text { del } \\
\text { Crecimiento }\end{array}$ & $\begin{array}{l}\text { Donella } \\
\text { Meadows, } \\
\text { Dennis } \\
\text { Meadows, } \\
\text { Jorgen } \\
\text { Randers. }\end{array}$ & $\begin{array}{l}\text { En la cual, con base a los datos recolectados desde } \\
\text { entonces, se exponía que la humanidad ya había } \\
\text { superado la capacidad de carga del planeta para } \\
\text { sostener su población. }\end{array}$ \\
\hline 1992 & $\begin{array}{l}\text { Programa o } \\
\text { Agenda } \\
\text { XXI }\end{array}$ & $\begin{array}{l}\text { Cumbre de } \\
\text { Rio de } \\
\text { Janeiro }\end{array}$ & $\begin{array}{l}\text { Documento que incluye acciones ambientales, que } \\
\text { deben ser adoptadas por las naciones a comienzos del } \\
\text { siglo XXI. Formulada en la Cumbre de Río y que } \\
\text { habría de formular un estrategia global sobre el destino } \\
\text { de la nave Tierra. }\end{array}$ \\
\hline 1992 & $\begin{array}{l}\text { Cambiando } \\
\text { el Rumbo }\end{array}$ & $\begin{array}{l}\text { Consejo } \\
\text { Empresari } \\
\text { al para el } \\
\text { Desarrollo } \\
\text { Sostenible }\end{array}$ & $\begin{array}{l}\text { Se establece un Sistema de mercados abiertos y } \\
\text { competitivos en los cuales los precios reflejan tanto los } \\
\text { costos del medio ambiente como los de otros recursos } \\
\text { (Stephan Schmidheiny, líder de la revolución } \\
\text { ambiental en el medio empresarial). }\end{array}$ \\
\hline 1992 & $\begin{array}{l}\text { Nuestra } \\
\text { Propia } \\
\text { Agenda }\end{array}$ & & $\begin{array}{l}\text { Informe auspiciado por el PNUD (Programa de las } \\
\text { Naciones Unidad para el Desarrollo), y el Banco } \\
\text { Interamericano de Desarrollo (BID) }\end{array}$ \\
\hline 2004 & $\begin{array}{l}\text { Los límites } \\
\text { del } \\
\text { crecimiento: } \\
30 \quad \text { años } \\
\text { después }\end{array}$ & $\begin{array}{l}\text { Dennis } \\
\text { Meadows, } \\
\text { Jorgen } \\
\text { Randers, } \\
\text { Donella } \\
\text { Meadows, }\end{array}$ & $\begin{array}{l}\text { Fue publicada el } 1 \text { de junio del } 2004 \text { por la "Chelsea } \\
\text { Green Publishing Company", en la que se actualizan e } \\
\text { integran las dos versiones precedentes. }\end{array}$ \\
\hline 2005 & $\begin{array}{l}\text { Informe de } \\
\text { Evaluación } \\
\text { de los } \\
\text { Ecosistemas } \\
\text { del Milenio, }\end{array}$ & $\begin{array}{l}\text { (Reid et } \\
\text { al,) }\end{array}$ & $\begin{array}{l}\text { Del cual se desprenden dos conclusiones } \\
\text { fundamentales: Primera en la segunda mitad del siglo } \\
\text { XX los ecosistemas se han deteriorado a una velocidad } \\
\text { no conocida en ningún otro período de la historia, } \\
\text { dando lugar a daños irreversibles. Segunda: Los } \\
\text { cambios producidos no son lineales y están } \\
\text { provocando el agravamiento de la pobreza de una gran } \\
\text { parte de la humanidad, por la destrucción de sus } \\
\text { formas tradicionales de vida.(Redondo, 2010) }\end{array}$ \\
\hline
\end{tabular}




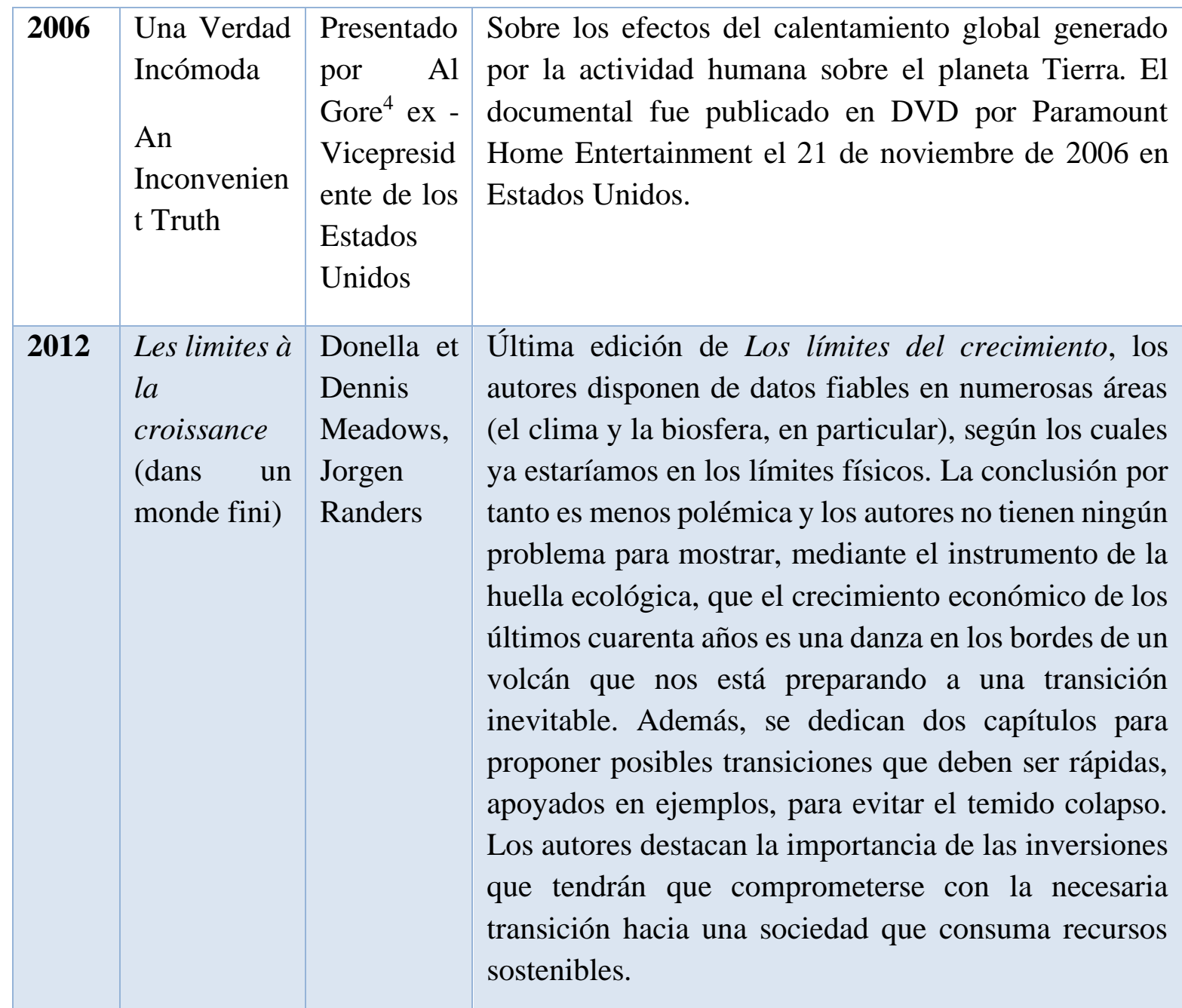

Fuente: elaboración propia

\section{Institucionalismo}

La primer conferencia registrada en la historia sobre la protección de la Naturaleza fue, en 1913 en Berna, Suiza convocada por Sarazin, presidente de la Liga Suiza para la Protección de la Naturaleza, que se cristalizaría años más tarde en 1928 cuando entra en funcionamiento la Oficina Internacional para la Protección de la Naturaleza y que para 1948 en Fointenebleau, Francia bajo los auspicios de la UNESCO, se crea y consolida como la Unión Internacional para la Protección de la Naturaleza y de sus Recursos (UIPN)

4 Por la autoría de este documental, Al Gore obtuvo el Premio Nobel de la Paz_en octubre de 2007, premio que comparte con el Grupo Intergubernamental de Expertos sobre el Cambio Climático (IPCC, por sus siglas en inglés) de Naciones Unidas. Al Gore ya había ganado en 2007 el Premio Príncipe de Asturias de Cooperación Internacional, así como el Oscar en 2006 a Mejor Documental y Mejor Canción Original para I Need to Wake Up. 
organización independiente que en la actualidad agrupa a centenares de otras organizaciones públicas y privadas. En el seno de su fundación T. Pritchar acuña la expresión Environmetal Education, haciendo referencia al enfoque educativo catalizador de una síntesis entre las ciencias naturales y sociales. En 1956 Cambia de nombre la UIPN a UICN, para convertirse en la Unión Internacional para la Conservación de la Naturaleza y los Recursos Naturaleza, con el objeto de fomentar la educación y difusión de la información relativa al medio ambiente.

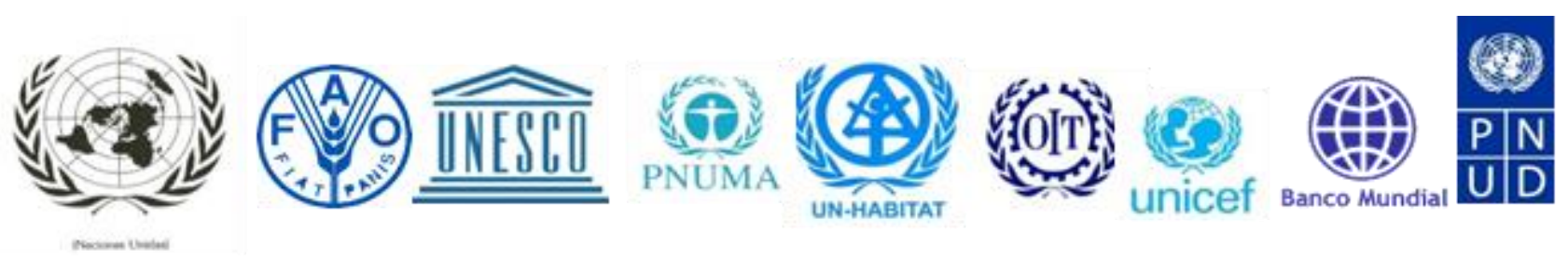

Figura 1: instituciones implicadas

A inicios de siglo XX el deseo de salvaguardar los múltiples espacios naturales y especies salvajes en peligro de desaparición, estimuló la creación de las principales organizaciones para proteger la naturaleza, que se fundaron durante la primera mitad del siglo XX. Entre ellas destaca el National Trust británico, organizado al estilo de una fundación, que ha logrado salvar infinidad de parajes naturales del Reino Unido.

Aun cuando la II Guerra Mundial estaba lejos de terminar, los países ya se preguntan ya sobre la manera en que iban a reconstruir los sistemas educativos una vez restablecida la paz. Muy rápidamente este proyecto crece y adquiere una dimensión universal. Nuevos gobiernos deciden participar, entre ellos el de los Estados Unidos de América.

Las guerras mundiales no solo postergaron y destrozan el proceso de internacionalización del movimiento de protección a la naturaleza de Berna, no obstante, se celebra nuevamente una conferencia en Basilea ${ }^{5}$, con esta temática y constituye el antecedente más claro de la primera organización mundial de conservación la UIPN antes mencionada.

Al finalizar la segunda guerra mundial con la firma de la Carta de las Naciones Unidas la Organización de las Naciones Unidas (ONU) fue fundada el 24 de octubre de 1945, en San Francisco (California), por 51 países, siendo actualmente la mayor organización internacional existente. La ONU se define como una asociación de gobierno global que facilita la cooperación en asuntos como el derecho internacional, la paz y seguridad internacional, el desarrollo económico y social, los asuntos humanitarios y los derechos humanos. En el mismo año como primera acción constituye la Organización de las Naciones Unidas para la Alimentación y la Agricultura, Por un mundo sin hambre. FAO, consecuentemente establece un organismo especializado UNESCO, por sus siglas en inglés (Organización de las Naciones Unidas para la Educación, la Ciencia y la Cultura) con el objetivo de contribuir a la paz y a la seguridad en el mundo mediante la educación, la ciencia,

${ }^{5}$ Ciudad suiza ubicada en la frontera con Francia y Alemania. 
la cultura y las comunicaciones. Ya entrada en funciones para 1948 la ONU integra la UIPN que como se menciona en el apartado anterior para 1956 se transformara en UICN, así mismo se constituye en 1961 la Organización para la Cooperación y el Desarrollo Económicos (OCDE) que agrupara 34 países miembros con la misión de promover políticas que mejoren el bienestar económico y social de las personas alrededor del mundo. En 1965 se genera el Programa de las Naciones Unidas para el Desarrollo (PNUD) y al siguiente año (1966) la Organización de las Naciones Unidas para el Desarrollo Industrial, (ONUDI), en 1968, se conforma el Club de Roma, por un pequeño grupo de científicos y políticos, que provenían de distintos países, invitados por el italiano Aurelio Peccei y el escocés Alexander King, para hablar de los cambios que se estaban produciendo en el planeta por consecuencia de acciones humanas.

Para 1973 arranca el Programa de las Naciones Unidas para el medio Ambiente (PNUMA), vinculado a éste se constituye la fundación Habitat. En 1976 se llevó a cabo, en Vancouver, Canadá, la Conferencia de las Naciones Unidas sobre Asentamientos Humanos (Hábitat I). En ese evento la Fundación Hábitat cambió de nombre y se transformó en el Centro de las Naciones Unidas para los Asentamientos Humanos (CNUAH), con sede en Nairobi, Kenia. Veinte años después, se realizó la segunda Conferencia de las Naciones Unidas para los Asentamientos Humanos (Hábitat II), en Estambul, Turquía. En octubre de ese año, la Oficina Regional para América Latina y el Caribe (ROLAC) comenzó operaciones en la Región, desde Rio de Janeiro, Brasil. y en el 2002, por decisión de la Asamblea General, el CNUAH pasó a ser el Programa de las Naciones Unidas para los Asentamientos Humanos, ONU-Habitat, a partir de ese año en adelante, la Oficina Regional abrió representaciones nacionales en México, Colombia, Ecuador, Cuba y Costa Rica. recientemente el trabajo de la Agencia ha llegado a Bolivia, Guatemala y El Salvador.

Más tarde con la intención de apoyar los esfuerzos de América Latina y el Caribe para reducir la pobreza y la desigualdad cuyo objetivo es lograr el desarrollo de manera sostenible y respetuosa con el clima, se funda en 1959, el Banco Mundial (BID), la mayor fuente de financiamiento para el desarrollo de América Latina y el Caribe, cuyo programa de reformas en evolución busca aumentar impacto en el desarrollo de la región conjuntamente con: la CEPAL (Comisión Económica para América Latina y el Caribe), la UNIFEM, (Fondo de Desarrollo de las Naciones Unidas para la Mujer), el Fondo de Naciones Unidas para la Infancia.(UNICEF), el Fondo de Población de las Naciones Unidas (UNFPA), la, Oficina del Alto Comisionado de las Naciones Unidas para los Derechos Humanos (OANUDH), la Organización Internacional del Trabajo (OIT), la Organización Mundial de la Salud (OMS), la Oficina de las Naciones Unidas para el control de las Drogas y la Prevención del Delito (ONUDD), la Convención de las Naciones Unidas para la Lucha contra la Desertificación (UNCCD por sus siglas en inglés) y el Centro de Información de las Naciones Unidas (CINU). Como se puede observar los Organismos, los Programas, Comisiones y Fondos de la Institución ONU están enfocados al desarrollo económico y ambiental del mundo.

Con base a lo anterior se puede inferir que a la par de las preocupaciones por el medio ambiente fueron emergiendo instituciones al igual que las publicaciones tanto de nivel global 
como local y que en consecuencia originara instituciones en cada nación, México no fue la excepción, a lo largo de cuatro décadas (1940-1980), la estrategia de desarrollo nacional se centró en el impulso a la industrialización a través de la sustitución de importaciones. El medio fundamental fue la intervención directa del Estado en la economía, que incluía la protección de un mercado interno.

Es a partir de 1971 que el desbordamiento de la problemática ambiental del país generó la necesidad de crear normas y reglamentos con la intención de frenar esa dinámica. La respuesta del Sector Oficial fue a través no solo de cambios legislativos sino en la propia restructuración de administración pública en sus tres ámbitos de gobierno, obedeciendo en gran parte al efecto de la relación de la salud con la problemática ambiental.

Tabla 2:Evolución institucional en el marco de la salud y el medio ambiente en México

\begin{tabular}{|c|c|}
\hline Institucional & Marco legal \\
\hline & $\begin{array}{l}\text { El antecedente más antiguo en materia de } \\
\text { regulación de medio ambiente en México data de } \\
1940 \text { Ley de Conservación de Suelos y Agua }\end{array}$ \\
\hline \multicolumn{2}{|l|}{1842 Dirección General de Industria } \\
\hline \multicolumn{2}{|l|}{$\begin{array}{l}1946 \text { Secretaría de Agricultura y Fomento. } \\
1946 \text { Secretaría de Recursos Hidráulicos que } \\
\text { en } 1972 \text { promulga la Ley Federal de Aguas. }\end{array}$} \\
\hline \multicolumn{2}{|l|}{$\begin{array}{l}\text { 1946-1976 Secretaría de Agricultura y } \\
\text { Ganadería. }\end{array}$} \\
\hline & $\begin{array}{l}\text { El } 23 \text { de marzo de 1971, se publica, la Ley para } \\
\text { Prevenir y Controlar la Contaminación } \\
\text { Ambiental, esta ley no tuvo el impacto esperado } \\
\text { y su tambaleante seguimiento no se tradujo en un } \\
\text { mejoramiento de las condiciones en las que se } \\
\text { encontraba el país en materia ambiental. }\end{array}$ \\
\hline $\begin{array}{lll}\text { 1972-1978 Subsecretaría para el } \\
\text { Mejoramiento del Ambiente dentro de la } \\
\text { Secretaría de Salubridad y Asistencia SSA. }\end{array}$ & $\begin{array}{l}1972 \text { Aparece el Plan Nacional Hidráulico, o } \\
\text { Ley Federal de Aguas, generado por la Secretaría } \\
\text { de Recursos Hidráulicos, con el que se } \\
\text { compromete con la Secretaría de Agricultura a la } \\
\text { regulación, inspección y vigilancia de la calidad } \\
\text { del agua en México. }\end{array}$ \\
\hline
\end{tabular}


1973 Se amplía el concepto saneamiento por el de Saneamiento Ambiental y se relaciona con actividades del mejoramiento y conservación del ambiente para preservar la salud y prevenir y controlar aquellas condiciones del ambiente que perjudican la salud humana (Branes, 1987).

1976-1994 creada la Secretaría de Agricultura y Recursos Hidráulicos SARH por el presidente José López Portillo para sustituir a la Secretaría de Recursos Hidráulicos, incorporando además la materia agraria que venía ejerciendo la Secretaría de Agricultura y Ganadería. Durante su existencia fue la encargada del aprovechamiento y conservación del agua.

1978 Desaparece la Subsecretaría de Mejoramiento del Ambiente y se crea la Dirección General de Ecología, dentro de la Secretaría de Asentamientos Humanos y Obras Públicas.

1982-1992 Se crea la Secretaría de Desarrollo Urbano y Ecología (SEDUE) con las atribuciones prevención y el control de contaminantes (agua, aire y suelos) y residuos peligrosos, preservación de recursos naturales y prevención de riesgos ambientales, de obras públicas y privadas, así como la integración del ordenamiento ecológico del territorio.

A la SEDUE estaba adscrita la Subsecretaría de Ecología, encargada de establecer la política ambiental en general, como a la emisión de reglamentos, normas y programas de acción.
En diciembre de 1976 con la asunción a la presidencia de José López Portillo y Rojas, se aprobó una nueva Ley Orgánica de la Administración Pública Federal, cuya exposición de motivos explica el cambio y la integración que sufrió la Secretaría de Recursos Hidráulicos.

1978 Con el retroceso de Subsecretaría a Dirección General, se pierden muchos recursos humanos y materiales y por ende, campo de acción y representatividad en el territorio mexicano.

1982 Por primera vez se incluye la temática ambiental, en el Plan Nacional de Desarrollo, este documento tuvo también poco impacto en el país, por la forma tan globalizada de abordar la problemática de la protección ambiental. El 11 de enero de 1982, se publicó la Ley Federal de Protección al Ambiente, con la finalidad de establecer de forma definitiva bases más firmes para la legislación ambiental vigente. A la SSA se le asigna la regulación y el control sanitario en materia de salud ambiental ocupacional, y saneamiento básico. (Estrada 1987)

1984 Se emite la Ley General de Salud, modificando el concepto que relaciona Salud y medio ambiente, a la concentración excesiva de contaminantes en el medio ambiente, uso y 


\begin{tabular}{|c|c|}
\hline & $\begin{array}{l}\text { consumo de agua, incluyendo aguas residuales; } \\
\text { saneamiento básico; ingeniería sanitaria; control } \\
\text { sanitario de las vías generales de comunicación; } \\
\text { de productos industriales gaseosos de alta } \\
\text { peligrosidad y fuentes de radiación. Se faculta a la } \\
\text { SSA para realizar otras actividades en las que se } \\
\text { representen situaciones que causen riesgos o } \\
\text { daños a la salud de la población (Brañes, 1987). } \\
\text { Posteriormente se presenta un proceso de } \\
\text { confusión y emisiones jurídicas y reglamentarias } \\
\text { donde la SSA pierde gran parte de sus } \\
\text { atribuciones con relación a la protección de la } \\
\text { salud ante riesgos ambientales. El proceso de } \\
\text { recuperación de las funciones ambientales de la } \\
\text { SSA se lleva a cabo a partir de las reformas de la } \\
\text { Ley General de Salud. }\end{array}$ \\
\hline \multicolumn{2}{|l|}{$\begin{array}{l}1987 \text { Se faculta al Congreso de la Unión para } \\
\text { legislar en materia de protección del ambiente }\end{array}$} \\
\hline & $\begin{array}{l}1988 \text { Se Reforma a la Constitución política con el } \\
\text { fin de preservar y restaurar el equilibrio ecológico } \\
\text { y aparece la Ley General del Equilibrio } \\
\text { Ecológico y Protección al Ambiente (LGEEPA) }\end{array}$ \\
\hline \multirow[t]{2}{*}{$\begin{array}{l}1989 \text { (CNA) Comisión Nacional del Agua, La } \\
\text { SSa en coordinación con SEDUE y la } \\
\text { Secretaría de Minas e Industria Paraestatal, } \\
\text { son responsables de la emisión y de las normas } \\
\text { técnicas para el uso, manejo almacenamiento, } \\
\text { distribución y disposición de los } \\
\text { contaminantes biológicos, químicos o físicos } \\
\text { que se consideran de alto riesgo para la salud } \\
\text { (diario oficial, 1989). En Jalisco se creó el } 31 \\
\text { de mayo, la Comisión Estatal de Ecología. }\end{array}$} & \\
\hline & $\begin{array}{l}\text { 1990 Para el mes de julio, se publicó el Programa } \\
\text { Nacional para la Protección del Medio } \\
\text { Ambiente en donde sobresale el reforzamiento } \\
\text { del marco legal en materia ambiental. }\end{array}$ \\
\hline
\end{tabular}




1992 Desaparece la SEDUE para
transformarse en la Secretaría de Desarrollo
Social, SEDESOL, dentro de la cual
aparecieron dos años más tarde en 1994 el
Instituto Nacional de Ecología (INE) y la
Procuraduría Federal de Protección al
Ambiente (PROFEPA) asegurando con ello
un desarrollo importante en el cumplimiento
de la normatividad ambiental aunque con sus
limitantes

La SARH Existió hasta 1994 cuando cambió su denominación por Secretaría de Agricultura, Ganadería y Desarrollo Rural. La moderna Comisión Nacional del Agua, CONAGUA nació como un organismo desconcentrado de esta secretaría.

1994 Desaparece la Secretaría de Agricultura y Recursos Hidráulicos SARH para transformarse el 18 de diciembre en (SEMARNAP) Secretaría de Medio Ambiente, Recursos Naturales y Pesca quien asume las funciones de la SEDESOL en lo relacionado a la administración y gestión ambiental y planea el manejo de recursos y políticas ambientales.

1995 En lo que al ámbito municipal se refiere no fue sino hasta 1995 cuando se creó la Dirección General de Medio Ambiente y Ecología, a la cual pertenecen: las Direcciones de Parques y Jardines; Mejoramiento Urbano; Manejo de Residuos y Prevención; y Control Ambiental, con lo que se da una atención a todo lo relacionado con la gestión y la protección ambiental, en el municipio de Guadalajara.

2000 la Secretaría del Medio Ambiente, Recursos Naturales y Pesca (SEMARNAP) cambia por Secretaría del Medio Ambiente,
1995 el 29 de diciembre se publicó el Reglamento Orgánico del Municipio de Guadalajara

2000 se reforma y adiciona la Ley Orgánica de la Administración Pública Federal dando origen a la Administración Pública Federal dando origen a la 


\begin{tabular}{|l|l|}
\hline $\begin{array}{l}\text { Recursos Naturales (SEMARNAT) así } \\
\text { mismo la Secretaria de Agricultura, Ganadería }\end{array}$ & $\begin{array}{l}\text { Secretaría de Medio Ambiente y Recursos } \\
\text { Naturales (SEMARNAT) }\end{array}$ \\
$\begin{array}{l}\text { y desarrollo Rural. Cambia a Secretaría de } \\
\text { Agricultura, Ganadería, Desarrollo Rural, } \\
\text { Pesca y Alimentación (SAGARPA) }\end{array}$ & \\
\hline & $\begin{array}{l}\text { 2004 se promulga Ley Regulatoria de Gestión de } \\
\text { Residuos peligrosos }\end{array}$ \\
\hline
\end{tabular}

De acuerdo a lo anterior tan solo el seguimiento de la metamorfosis de una secretaría (SAGARPA1842-2000) permite inferir la influencia de la transición del desarrollo, así como de la creación de nuevas instituciones de nivel global. Todos estos cambios sufridos en las estructuras de regulación gubernamental federales, desafortunadamente han entorpecido la continuidad a los programas de protección y conservación, reflejando principalmente un desgaste de recursos económico, humano y hasta ecológico que se reflejan en el desarrollo del país y sus actividades productivas.

Esta ha sido la forma en la que las instancias gubernamentales enfrentan el problema del deterioro ambiental, como se ha observado en la tabla anterior, la salud ambiental originalmente se enfocaba a los aspectos relacionados con la higiene de los alimentos, saneamiento básico e higiene ocupacional, una vez reconocido el impacto ambiental a la salud por la exposición a sustancias tóxicas y otros factores del entorno, el concepto de salud ambiental ha sido modificado.

\section{El panorama del desarrollo}

Pareciera que el fútil debate semántico entre términos como sustentable ${ }^{6}$ y sostenible que resurgieran en la década de los 70s fuera más relevante que la de aclarar el papel de cada individuo en su entorno, como parte y no como "dueño" de él. Todavía en los medios se pueden leer exhortaciones institucionales para "cuidar el medio ambiente" o "salvar al medio ambiente" pero si nos preguntamos ¿cuidarlo de qué? o ¿salvarlo de quién? la respuesta siempre es: "de nosotros mismos". Se dice pues cuidar o salvar cuando lo que se debe decir es respetar objetivo que incluye el manejo responsable (respetuoso y consciente), por lo tanto al medio ambiente no se le protege, se le aprovecha a través de un manejo inteligente. Ya que "él mismo" es quien se regula y se equilibra porque su inherente resiliencia.

La humanidad ha adoptado definiciones como la de desarrollo sustentable que de acuerdo al Reporte Brundtland lo "define" como: "aquel que permite satisfacer nuestras necesidades actuales sin comprometer la capacidad de las generaciones futuras para satisfacer las

\footnotetext{
6 El concepto sustained-yield (rendimiento sustentado) una traducción al inglés del término germano nachhaltigkei (mantener para después).
} 
suyas". ${ }^{7}$ definición que no dice nada porque ni define el desarrollo ni lo que es sustentable, menciona solamente un principio que deben considerar objetivos, pero no aclara que el desarrollo sustentable no es un puerto o destino al que hay que llegar como se piensa en la economía, sino un "rumbo" que nos señala a todos por donde conducirnos. Los conceptos medio ambiente y desarrollo sustentable han sido hilvanados por la historia, el medio ambiente es considerado como la urdimbre para el desarrollo sea este sustentable o no, la educación ambiental como la mano del tejedor (el hombre) motor o herramienta que debe tejer los hilos a través del aprovechamiento consciente, mesurado, respetuoso, de los recursos, hilo sujeto a su lanzadera (políticas, tecnología, economía...) cuyo proceso teja este lienzo llamado desarrollo. El debate por el medio ambiente es en el que la humanidad siempre es juez y parte, pero donde el implicado "medio ambiente" nunca está invitado y donde nunca aplica el principio jurídico In dubio pro reo ${ }^{8}$

Para entender el concepto medio ambiente es necesario recorrer el desarrollo humano desde una perspectiva no solo social sino económica, política, tecnológica y ambiental. Inicialmente el desarrollo humano estaba orientado a la sobrevivencia donde el medio ambiente fungía como escenario, conforme se fueron dando los primeros asentamientos humanos, el desarrollo se reorienta a la conquista del espacio y el medio ambiente pasa a ser un botín. La ciencia los descubrimientos ${ }^{9}$ los inventos y la investigación son precursores del impulso al desarrollo que por un lado incrementa de forma exponencial la población humana y en consecuencia y por otro lado el aumento de la demanda de recursos naturales (agua, alimento, energía, suelo), evidenciando la distinción entre los renovables y los no renovables, y nuevamente la percepción del ambiente toma un nuevo rumo como el Stock de la humanidad. Es hasta después de la Revolución Industrial en la que la alta demanda de recursos para su explotación propiciada por el aumento de la población mundial y del desarrollo tecnológico, que se evidencia la devastación de los bosques y propicia las primeras reflexiones.

Al final de siglo XIX el movimiento conservacionista estadounidense surgió cuando varios ciudadanos y funcionarios del gobierno empezaron a darse cuenta de la magnitud de la deforestación y el agotamiento de la vida silvestre en todo el país. La acción federal en la conservación de los recursos forestales y la vida silvestre empezó en 1872 cuando el gobierno

En el año 1987 se publicó un reporte llamado "Nuestro Futuro Común" que tuvo un gran impacto en la historia del movimiento ambientalista. El Informe Brundtland, como también se lo conoce, analizó la situación del mundo en ese momento y demostró que el camino que la sociedad global había tomado estaba destruyendo el ambiente por un lado y dejando a cada vez más gente en la pobreza y la vulnerabilidad. Viendo este problema se hizo una pregunta muy importante: ¿Cómo podrá este estilo de desarrollo servir en el siglo próximo XXI si el mundo estará poblado por el doble de personas, todas dependiendo del mismo ambiente? La respuesta fue que no se podía seguir con el crecimiento económico tradicional y que había que buscar un nuevo estilo de desarrollo al que llamó sustentable, éste debía ser más justo y equitativo y permitir a la humanidad satisfacer sus necesidades sin comprometer la capacidad de las futuras generaciones de satisfacer las suyas.

8 In dubio pro reo (en caso de duda a favor del acusado) principio jurídico donde el fiscal o agente estatal equivalente debe probar la culpa del acusado y no este último su inocencia. que tiene su propio relato, pero sobre todo en la apelación.

91981 Científicos británicos anuncian que desde 1970 se reproduce, cada primavera, un agujero en la capa de ozono en la Antártida, provocado por la acción de los CFC (gases clorofluorcarbonados). En 1990 se confirma que otro agujero se produce en el polo Norte. 
protegió 809.000 ha de bosque, principalmente en el noroeste de Wyoming, y prohibió la cacería en esa región, es en ese mismo año que se declara en Estados Unidos el Primer Parque Nacional "Yellowstone". No obstante, la percepción de bonanza de recursos naturales inspira acciones y hábitos de desperdicio y ultraje de recursos, en 1900 como primer esfuerzo internacional a favor de la conservación para revertir tales hábitos negativos, se realiza en África la Primer Convención para la preservación de la vida salvaje y aunque sus recomendaciones como en el Derecho aplicado en las Indias españolas "’'Obedézcase pero no se cumpla" ${ }^{10}$ no fueron aplicadas, sus decisiones influyeron en la organización de reservas de caza y constituyen un precedente de la toma de conciencia global, que fuera postergada por el by pass de las Guerras Mundiales, es hasta la posguerra 1945, se funda la Organización de las Naciones Unidas, (ONU) dado los quebrantables resultados de los bombardeos los suelos productivos también habían sido prácticamente exterminados, por lo que en sus primeras iniciativas forma el 16 de octubre la Fundación para la Agricultura y la Alimentación (FAO), Iniciada la postguerra donde el medio amiente pasa a ser el protagonista principal en el escenario del desarrollo y es observado éste como el personaje principal postura que cambia la percepción social del ambiente.

El sinnúmero de eventos que habían venido dándose desde el preludio del desarrollo humano, tanto los desastres naturales (temblores, erupciones volcánicas, tornados, ciclones, tormentas...) aunado a los desastres antropogénicos (catástrofes, guerras, epidemias, desertificación, sequía, hambruna, pobreza...) tienen un solo protagonista la vorágine ambición humana, no tardaron en resurgir las instituciones en las que a raíz de la segunda guerra mundial pretendieron paliar la devastadora debacle.

La Organización de las Naciones Unidas (ver apartado Institucionalismo) que aglutinara una postura global para la solución organizada de los problemas emergentes continua generando programas, fundaciones y organismo como la el Fondo Internacional de Desarrollo Agrícola $\left(\right.$ FIDA $^{11}$ ), la Organización para la Cooperación y Desarrollo Económicos (OCDE), la Organización de las Naciones Unidas para el Desarrollo Industrial (ONUDI), para atender problemas de alimentación y desarrollo, en consecuencia a los problemas ambientales se constituye la Unión Internacional para la Conservación de la Naturaleza (UICN) el Programa de las Naciones Unidas para el Desarrollo (PNUD) y el Programa de las Naciones unidas para el Medio Ambiente (PNUMA), con enfoque a los recursos el medio ambiente y de forma complementaria aparece la UNESCO.

\footnotetext{
${ }^{10}$ La figura jurídica del obedézcase pero no se cumpla, propia, típica y endémica del Derecho Histórico Castellano, y profusamente presente en el Derecho aplicado en las Indias españolas, nació en el siglo XIV (Cortes de Burgos y Briviesca de 1379 y 1387) y se generalizó su uso durante siglos como medio válido para dejar de aplicar aquellas normas que, pese a haber sido promulgadas por autoridad legítima, frecuentemente el Rey, suponían una vulneración de las leyes o fueros propios, normas, usos o costumbres del lugar. De esta manera la Auctoritas del Rey quedaba salvaguardada, y las normas del lugar, que allí se consideraban justas, seguían rigiendo con plena eficacia.

Tomado de http://www.garrigues.com/es/publicaciones/articulos/Paginas/Obedezcase-pero-no-se-cumpla.aspx

11 FIDA Agencia especializada de las Naciones Unidas dedicada a promocionar el progreso económico de los habitantes pobres de zonas rurales.
} 
De este institucionalismo global se derivan otro maremágnum comisiones y organismos que pretenden trabajar en cada una de las naciones. La ONU forma grupos y asociaciones como el Club de Roma, el MAB, quienes bajo formatos de acuerdos agentas, cartas, convenios reuniones cumbre declaraciones, movimiento, reportes y protocolos, intentan dar directrices al desarrollo en temáticas de abordaje como la protección forestal, bonos carbono, conservación, planes de acción, multidisciplinariedad, declaración de décadas y años de acción, educación ambiental que luego se convirtiera en educación para el desarrollo sustentable, los estudios y evaluaciones de impacto ambiental, movimientos ecologistas, el ordenamiento territorial, programas ambientales y la rehabilitación. Todos dirigidos a solucionar los factores causales del deterioro ambiental como la pobreza, la falta de respeto a los derechos humanos, falta de vivienda digna, abuso del recurso, educación insuficiente entre otros.

Para la toma de decisiones la búsqueda espuria de la soberanía ambiental había llevado por el proceso correcto en la búsqueda de ¿quiénes somos? a ¿dónde vamos? ambientalmente, tropezando lamentablemente con saraos y cantos de victoria precipitados de los pequeños logros. La lisonja de aplausos y reconocimientos nublaron la meta: equilibrio entre el hombre con el hombre y el hombre con la naturaleza.

Valdría la pena preguntarse ¿Qué sigue en esta novela? quizá la tecnología estará buscando la solución al deterioro ambiental como una aplicación al celular, la economía pugnará por la mejor distribución de la riqueza, la política seguirá generando nuevos instrumentos de regulación, las ciencias encontrarán nuevas explicaciones, y la sociedad seguirá aglutinándose para hacer actos de contrición para generar nuevas penitencias. Pero aquí la pregunta más importante queda en el tintero, ¿Qué estoy haciendo yo ante el deterioro ambiental? aunque pareciera quehacer solo para expertos ambientalistas, es tarea de todos ya que, sin pretender una postura reduccionista, el deterioro ambiental es el resultado de la suma de nuestros hábitos de consumo por lo que la respuesta correcta no requiere de sofisticación es tan simple como: conocer, minimizar, compartir, ahorrar, revertir, actuar, reducir, reciclar, rechazar.

\section{Referencias bibliográficas}

Brañez R., (1987) Derecho Ambiental Mexicano. México: Fundación Universo XXI

Covarrubias T. N. (2000). Estrategia de Educación Ambiental Urbana, Tesis de maestría en Educación Ambiental, Universidad de Guadalajara, Jalisco, México.

Estrada O. S. (1989) Medio Ambiente y Salud. México: Fundación Universo XXI 
Historia Universal (2014) La era de las revoluciones, en Sites, recuperado el 8 de agosto 2014 de sitio https://sites.google.com/site/librohistoriauniversal/la-era-de-lasrevoluciones

"Los ímites del crecimiento" (2014) recuperado el 5 de agosto 2014 http://es.wikipedia.org/wiki/Los_1\%C3\%ADmites_del_crecimiento

Redondo G. L. (2010) Vigencia del Manifiesto ecologista para la supervivencia, Boletín $C F+S, \quad 46, \quad$ pp. 39-42. Recurso electrónico en línea: http://habitat.aq.upm.es/boletin/n46/alred.html [Consulta: 11 de agosto de 2014]

Urquidi V. L. (2007) Desarrollo sustentable y Cambio Global, Obras escogidas de Víctor L Urquidi., México: Ed. Alejandro Nadal, Colegio de México. 